\title{
The Birmingham Registry for Twin and Heritability Studies (BiRTHS)
}

\author{
Ruth E. Krone, ${ }^{1,2}$ Andrew K. Ewer, ${ }^{2}$ Timothy G. Barrett, ${ }^{3}$ Robert J. Moy, ${ }^{3}$ Shagaf Bakour, ${ }^{4}$ Eamonn R. Maher, ${ }^{5,8}$ \\ Shakila Thangaratinam, ${ }^{6}$ Philip Cox, ${ }^{7}$ Bill Martin, ${ }^{6}$ Khalid S. Khan, ${ }^{6,8}$ and Maurice P. Zeegers ${ }^{1}$ \\ ' Unit of Genetic Epidemiology, Department of Public Health and Epidemiology, University of Birmingham, Birmingham, United Kingdom \\ ${ }^{2}$ Neonatal Unit, Birmingham Women's Hospital, Edgbaston , Birmingham, United Kingdom \\ ${ }^{3}$ Institute of Child Health, University of Birmingham, Birmingham, United Kingdom \\ ${ }^{4}$ City Hospital, Department of Obstetrics and Gynaecology, Birmingham, United Kingdom \\ ${ }^{5}$ Section of Medical and Molecular Genetics, Department of Paediatrics and Child Health, Medical School, University of Birmingham, Edgbaston, \\ Birmingham, United Kingdom \\ ${ }^{6}$ Department of Obstetrics, Birmingham Women's Hospital, Edgbaston, Birmingham, United Kingdom \\ ${ }^{7}$ Perinatal Pathology, Birmingham Women's Hospital, Edgbaston, Birmingham, United Kingdom \\ ${ }^{8}$ Department of Obstetrics and Gynecology, University of Birmingham, Edgbaston, Birmingham, United Kingdom
}

In this article we present the protocol of the Birmingham Registry for Twin Heritability Studies (BiRTHS), which aims to establish a long-term prospective twin registry with twins identified from the antenatal period and subjected to detailed follow-up. We plan to investigate the concordance in anthropometrics and early childhood phenotypes between 66 monozygotic and 154 dizygotic twin pairs in the first 2 years of recruitment. In this project we plan to determine the relative contributions of heritability and environment to fetal growth, birth size, growth in infancy and development up to 2 years of age in an ethnically mixed population. Twins will be assessed with the Griffitth's Mental Development Scales, which will enable us to obtain detailed information on development. As maternal depression may have an effect on the twins' neurodevelopment, the Edinburgh Postnatal Depression Scale will be used at various stages during pregnancy and after delivery to assess maternal depressive symptoms. The increasing prevalence of obesity in both adults and children has raised concerns about the effect of maternal obesity in pregnancy on fetal growth. The prospective study design gives us the opportunity to obtain data on maternal nutrition (reflected by body mass index) and ante- and postnatal growth and development of twins.

People of South Asian origin (encompassing India, Sri Lanka, Nepal, Pakistan, and Bangladesh) make up over $20 \%$ of the world population and are strongly predisposed to cardiovascular disease as well as development of insulin resistance and its metabolic abnormalities, which are known to preceed coronary heart disease (Dhawan et al., 1994). Compared to the White United Kingdom population, South Asian migrants experience a 1.5 to 4.0 times higher cardiovascular disease mortality (Enas et al., 1992) despite the fact that nearly half of this group are lifelong veg- etarians and the incidence of other classic risk factors is low (Enas \& Mehta, 1995). In this group cardiovascular disease has the tendency to occur early in life and to follow a malignant course (Enas \& Mehta, 1995).

It is now well established that the fetal environment plays a major role in determining metabolism in childhood and adulthood and that low birthweight increases the risk of developing type II diabetes, cardiovascular disease (Ong et al., 2000) and obesity (Hales et al., 1991; Valdez et al., 1994; Yarbrough et al., 1998).

There are two principal explanations for these associations. Barker suggests in his thrifty phenotype hypothesis that fetal undernutrition during critical periods of development forces fetal endocrine and metabolic adaptation with detrimental long-term effects (Barker \& Clark, 1997; Hales \& Barker, 1992). The second possible explanation is that the associations are due to the effect of genes that influence fetal growth to give a selective survival advantage in times of undernutrition, but have a longterm predisposition to adult disease (Neel, 1962; Prentice et al., 2005).

In Birmingham United Kingdom, around $43 \%$ of newborn babies and children under 15 years of age are from ethnic minority populations, mainly of Pakistani descent from the region of Mirpur (Census 2001). Asian babies contribute proportionately more to perinatal mortality than White babies, which is widely held to be associated with a higher incidence of fetal growth restriction (Chetcuti et al., 1985; Wilcox et al., 1993). The latter may be related to

Received 27 July, 2006; accepted 25 August, 2006.

Address for correspondence: Professor Maurice P. Zeegers, Unit of Genetic Epidemiology, Department of Public Health \& Epidemiology, University of Birmingham, Birmingham B15 2TT, UK. E-mail: m.p.zeegers@bham.ac.uk 
adverse environmental factors including maternal size, nutrition and health and also to inadequate antenatal care. All these factors may prevail over heritability in influencing birth size and early postnatal growth.

People of India have been shown to develop noninsulin-dependent diabetes about 11 years earlier and with a lower body mass index (BMI) than White Caucasians (Mukhopadhyay et al., 2006). They have a higher waist-to-hip ratio (Primatesta \& Hirani, 2001) and a higher percentage of body fat for a given BMI, but a reduced muscle mass, so the body composition is likely to be responsible for the insulin resistance of South Asians (McKeigue et al., 1991). Yajnik et al. (2002) have shown that adiposity and hyperinsulinaemia are present at birth. From their collected anthropometric data, they concluded that the thin but centrally fat phenotype of Indian adults (Banerji et al., 1999) originates during fetal life, underlined by the fact that both insulin and leptin levels measured in cord blood were higher in Indian than in White Caucasian term newborns when related to birthweight (Yajnik et al., 2002).

The rapidly increasing frequency of obesity in all age and ethnic groups has raised concerns about the potential effects of maternal obesity in pregnancy on fetal growth. Animal studies have shown that maternal overnutrition retards placental and fetal growth and therefore increases both fetal and neonatal mortality (Wallace et al., 2003). Maternal obesity in humans is also known to result in fetal growth restriction with increased mortality (Wu et al., 2004). In addition, maternal obesity might lead to an elevated risk of these mothers' offspring becoming obese themselves with early onset of all metabolic complications (Boney et al., 2005; Ray et al., 2001).
Among other things, a child's development is dependent on maternal health and psychological stability. For women, pregnancy and childbirth represent a time of great vulnerability to the development of depressive symptoms (Dennis, 2004), and 10\% to $15 \%$ of all mothers are affected by postnatal depression (Cooper et al., 1988; Gotlib et al., 1989; O’Hara et al., 1984). Impaired maternal health is known to have severe long-term effects for maternal-infant interactions, resulting in adverse outcomes for the child, such as attachment insecurity, emotional and social developmental delay (Cooper \& Murray, 1997; Dennis, 2004). Currently it is unclear to what extent these depressive symptoms might be apparent antenatally, and whether they can predict later onset of postpartum depression. Furthermore, the incidence of pregnancy-related depression and its effect on the offspring have not been investigated previously in a cohort of mothers expecting twins.

This unique setting has provided us the opportunity to investigate the heritability of growth in infancy and developmental parameters in an ethnically mixed population. This project is the first step in setting up the BiRTHS Twin Registry, which aims to establish a long-term prospective twin study with twins identified antenatally and undergoing detailed follow-up in various respects.

\section{Methods}

\section{Recruitment of Twins}

Twins will be recruited antenatally from three large hospitals in Birmingham, which is the second largest city in the United Kingdom. Taken together, these three hospitals have approximately 16,000 deliveries per year, including 200 pairs of twins. Upon confirmation

Table 1

Information about Maternal and Family Medical History, Taken from Pregnancy Notes

\begin{tabular}{|c|c|}
\hline Topic & Data obtained \\
\hline \multirow[t]{2}{*}{ Maternal personal details } & $\begin{array}{l}\text { Date of birth, age, height, weight and BMI kg/m² in early pregnancy } \\
\text { and at birth }\end{array}$ \\
\hline & Country of birth, ethnic origin \\
\hline Previous pregnancies & $\begin{array}{l}\text { Number, age and condition of children, birthweight, gestational age, } \\
\text { complications }\end{array}$ \\
\hline \multirow[t]{2}{*}{ Fathers details } & Date of birth, age \\
\hline & Blood related to mother? Country of birth, ethnic origin \\
\hline Socioeconomic status (both parents) & Employment, occupation \\
\hline \multirow[t]{2}{*}{ Maternal medical history } & Diabetes, endocrine disorders, cardiovascular disease \\
\hline & Fertility treatment \\
\hline Family medical history & $\begin{array}{l}\text { Diabetes, endocrine disorders, cardiovascular disease, family history } \\
\text { of genetic pathology, stillbirths or multiple miscarriages, } \\
\text { sudden infant death }\end{array}$ \\
\hline Ultrasound scan details & $\begin{array}{l}\text { Gestational age, presentation, biparietal diameter, head circumference, } \\
\text { femur length, estimated fetal weight, placenta, Amniotic Fluid }\end{array}$ \\
\hline \multirow[t]{2}{*}{ Smoking/alcohol/drugs } & Number of units per day \\
\hline & Medical program (e.g., Methadone) \\
\hline
\end{tabular}


Table 2

Details Taken at Birth

\begin{tabular}{|c|c|}
\hline Topic & Details \\
\hline \multirow[t]{3}{*}{ Anthropometric details } & Sex \\
\hline & Weight, crown-heel-length, occipitofrontal head circumference \\
\hline & Midarm circumference, subscapsular and triceps skin fold thickness \\
\hline \multirow[t]{3}{*}{ Delivery details } & Mode of delivery \\
\hline & Complications (fetal/maternal) \\
\hline & Presentation at birth (cephalic, breech, other) \\
\hline \multirow[t]{3}{*}{ Data reflecting the newborn's condition at birth } & Apgar score taken at 1 and 5 minutes \\
\hline & Cord gases \\
\hline & Resuscitation \\
\hline \multirow[t]{4}{*}{ Postnatal complications } & Ventilation: CPAP, intubation, oxygen \\
\hline & Infection \\
\hline & Respiratory distress syndrome \\
\hline & Hypoglycaemia, jaundice requiring phototherapy \\
\hline Mode of feeding & Breastfeed/bottle feed; tube feed, total parental nutrition \\
\hline Discharge & $\begin{array}{l}\text { Gestational age and date at discharge, number of days spent } \\
\text { on neonatal unit and /or transitional care ward }\end{array}$ \\
\hline
\end{tabular}

of twin pregnancy by routine ultrasound scan in the antenatal booking clinic (within first trimester), standard care is transferred to a specialized multiples clinic or clinician, where expectant twin parents will be recruited. As health care is free and equal for residents of the United Kingdom, similar routine care for the different ethnic groups is to be expected.

Once parents have given informed consent to take part in the study, the mother will be asked to fill out an antenatal questionnaire on a visit to the antenatal special multiples clinic (around 20-24 weeks of gestation). This questionnaire will cover twinning history in both parents' families, maternal depression and exercise. Information about maternal and family medical history will be collected using standardized pregnancy notes (Table 1).

All twins included in this study will meet the World Health Organization (WHO) criteria for liveborn infants (birthweight $\geq 500 \mathrm{~g}$ or gestational age $\geq$ 22 weeks, if birthweight unknown). Exclusion criteria are decline of consent, death and severe malformation (i.e., a malformation that is likely to cause death or severely impairs subsequent growth and development). Within the first 2 years we expect to recruit about 66 monozygotic (MZ) and 154 dizygotic (DZ) twin pairs, assuming a recruitment rate of $50 \%$.

After birth, data that are routinely obtained during standard care will be collected retrospectively. These data include sex, anthropometrics, details of delivery and postnatal course (see Table 2 above). In addition, midarm circumference, triceps and subscapular skin fold thickness will be measured within the first 3 days of life using a Holtain calliper.

All placentae will be collected, weighed and macroscopically examined to confirm chorionicity. The placentae will be stored at $-4{ }^{\circ} \mathrm{C}$ for subsequent genetic epidemiological research. Five $\mathrm{ml}$ of cord blood will be taken from each twin from the placental end of the cord, and DNA from all twins will be extracted.

\section{Determination of Zygosity}

Zygosity will be determined through sequential analyses based on sex, fetal membranes and genotyping. When zygosity cannot be inferred based on sex and chorionicity, 12 polymorphic DNA markers will be examined using one DNA aliquot. MZ twins will share the same pattern for all 12 markers. The microsatellite markers will be selected from the Research Genetics panel to provide a high degree of information in each of the ethnic groups to be tested. The twins' zygosity status will be communicated to the parents and registered in the BiRTHS database.

\section{Follow-Up of Twins}

After birth, parents will be invited to visit a designated multiples clinic 3-monthly during the first year of life and 6-monthly during the second. At these clinics, the twins' postnatal anthropometrics, phenotypical characteristics and development will be closely monitored. For the latter, the revised and validated Griffiths Mental Development Scales (Griffiths, 1976; Ramsay \& Fitzhardinge, 1977) for children 0 to 2 years will be used to assess developmental status annually. This assessment contains five separate scales: locomotor, personal-social, hearing and speech, eye and hand coordination, and performance. Detailed follow-up of anthropometric data - weight, length, growth velocity, BMI, circumference of head and midarm and skin fold thickness - will be taken at each visit.

For additional information, the parents will be asked to complete parent specific follow-up questionnaires. 


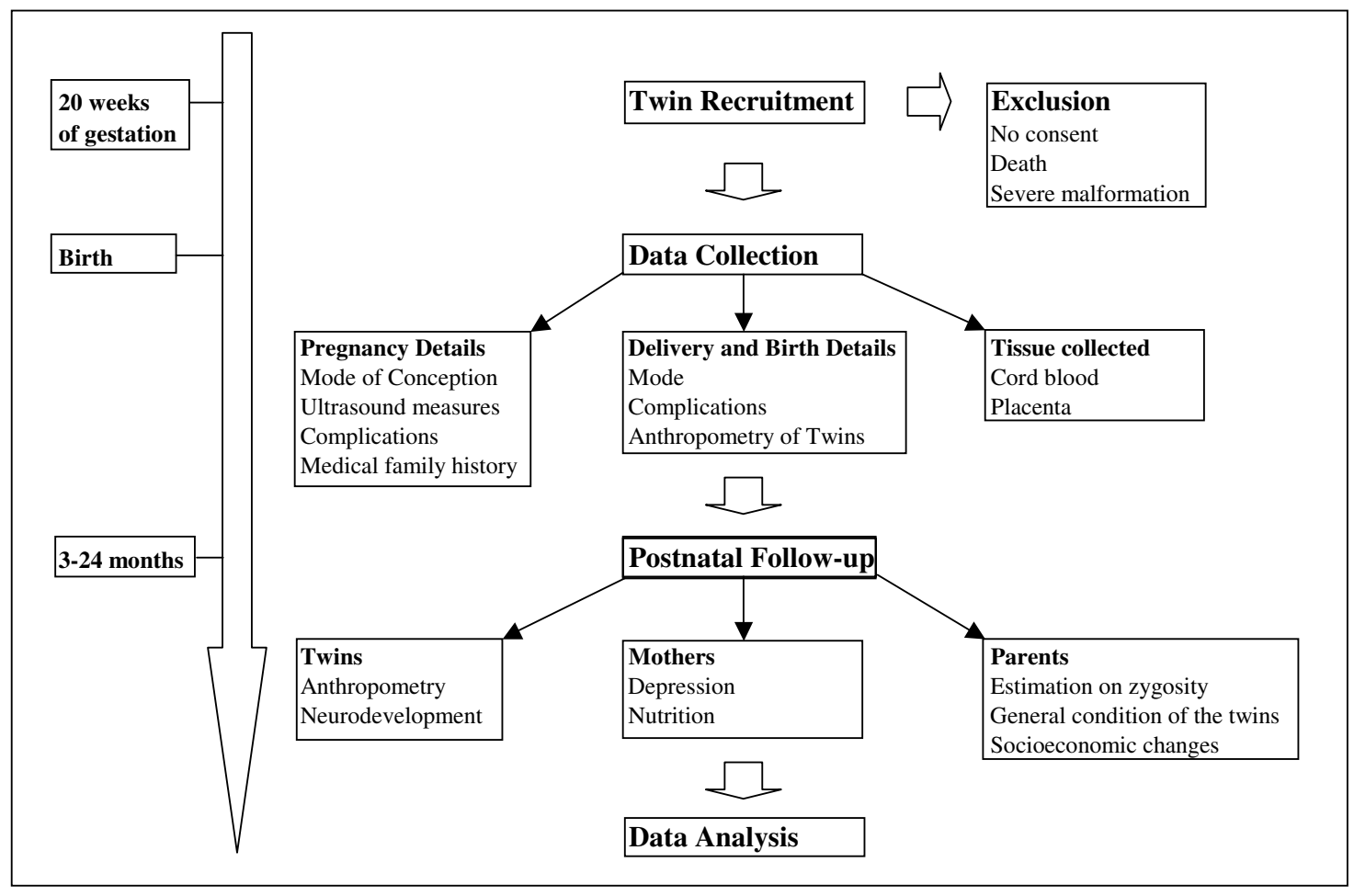

Figure 1

Time schedule.

These questionnaires will deal with the twins' general condition since birth, for example, patterns of sleeping, feeding and general health. They will be asked about their belief about zygosity and similarity of their twins. By use of the standardized child health record and interviewing the parents, developmental milestones will be assessed. Changes in socioeconomic status (e.g., loss of income due to maternity leave or mother stopping work) will also be obtained. Figure 1 shows detail and timing of all measurements to be taken in the first 2 years of life. All data will be entered into a password protected twin registry.

Using the Edinburgh Postnatal Depression Scale (EPDS; Cox et al., 1987), a 10-item self-report instrument, mothers will be screened for depression during and after pregnancy. Developed as a screening device, the EPDS has been shown to have a specificity of $92.5 \%$ and a sensitivity of $88 \%$ for detection of depressive symptoms in the general population (Murray \& Carothers, 1990). If depressive symptoms are detected, the general practitioner will be informed and appropriate care initiated.

\section{Statistical Analyses}

Differences in growth and pre- and postnatal depression scores and the effect of maternal nutrition (reflected by BMI) on postnatal growth will be evaluated by using multivariable linear regression analyses in which potential confounders will be incorporated as covariates in the regression model. Interaction models will estimate effect modification by ethnic group.

Heritability of all discrete and continuous phenotypes as described above will be estimated by univariate and multivariate variance components modelling using the structural equation modeling program Mx (Neale et al., 2002). Also potential interaction by sex or ethnic background will be evaluated to generate clues for prospective analyses.

\section{$\overline{\text { Discussion }}$}

The BiRTHS twin registry will be able to investigate the concordance in anthropometrics and early childhood phenotypes up to 2 years of age between $66 \mathrm{MZ}$ and $154 \mathrm{DZ}$ twins. It is anticipated that BiRTHS will be the basis for further follow-up to 10 years of life or even adulthood.

Our goal is to determine the relationship between parental characteristics and twinning as well as the relative contributions of heritability and environment to pre- and postnatal growth and development. Consequences of twinning for parents and twins will also be studied. The study's design will provide data to study Barker's hypothesis concerning long-term effects of intrauterine adverse environment. This project with its unique setting will provide clues for potential differences in heritability according to ethnic background. We hypothesize that the varying size and weight of babies of European and Asian origin is due to varying contribution of genes and environment. We predict 
that the concordance in birthweight gain between $\mathrm{MZ}$ Asian twins will be lesser than in their White peers, indicating a greater contribution of environmental factors to weight gain in Asians compared to White babies. We expect first results on growth and development after 2 years.

Using the Griffiths Mental Development Scales, the mental neurodevelopment will be monitored in detail as well. To our knowledge, no previous twin study has investigated both physical and mental development in this detail. It is now well established that the fetal and early postnatal period is crucial not only for growth, but for mental development as well (Tolsa et al., 2004). Recent studies have shown a trend to mild learning difficulties and difficulties in attention in children who were born small for gestational age (defined as < 10th percentile for gestational age and gender; O'Keeffe et al., 2003). Our prospective study design with detailed follow-up of both mental and physical development will give a greater understanding of the relationship between growth and neurodevelopment.

Parental questionnaires will reveal information about socioeconomic changes, behavior and maternal depressive symptoms and their implication for twin development. One interesting aspect is the parents' attitude towards their twins regarding zygosity and similarity and the change over time.

For the future we plan to link more centers in the West Midlands region to BiRTHS to establish a broad local twin registry. Many questions of interest will not be answered sufficiently after 2 years. Different patterns of long-term metabolic (development of type II diabetes and obesity), endocrine (precocious puberty or short stature in former small-for-gestational-age babies), cardiac (early onset of cardiac heart disease) and other medical disorders (increased risk of cancer) and their relation to intrauterine and early childhood growth within different ethnic groups can be investigated. Furthermore, as the development is monitored, we will be able to show relationships between growth and neurodevelopment.

The main strengths of our project are the antenatal recruitment strategy, the interdisciplinary data acquisition and the ethnically mixed population. BiRTHS will be a springboard for long-term follow-up of the effects of hostile intrauterine environment not only in medical, but also in psychosocial and socioeconomic respects.

\section{Acknowledgments}

This study is supported by the Birmingham Children's Hospital Research Foundation. We are grateful to Dorret Boomsma, Gargeswari Sunanda, Neil Shah, and Sabah Baghdadi for their valuable contribution to this paper and the study protocol.

\section{References}

Banerji, M. A., Faridi, N., Atluri, R., Chaiken, R. L., \& Lebovitz, H. E. (1999). Body composition, visceral fat, leptin, and insulin resistance in Asian Indian men.
Journal of Clinical Endocrinology and Metabolism, 84, 137-144.

Barker, D. J., \& Clark, P. M. (1997). Fetal undernutrition and disease in later life. Reviews of Reproduction, 2, 105-112.

Boney, C. M., Verma, A., Tucker, R., \& Vohr, B. R. (2005). Metabolic syndrome in childhood: Association with birth weight, maternal obesity, and gestational diabetes mellitus. Pediatrics, 115, e290-296.

Chetcuti, P., Sinha, S. H., \& Levene, M. I. (1985). Birth size in Indian ethnic subgroups born in Britain. Archives of Disease in Childhood, 60, 868-870.

Cooper, P., \& Murray, L. (1997). Prediction, detection, and treatment of postnatal depression. Archives of Disease in Childhood, 77, 97-99.

Cooper, P. J., Campbell, E. A., Day, A., Kennerley, H., \& Bond, A. (1988). Non-psychotic psychiatric disorder after childbirth. A prospective study of prevalence, incidence, course and nature. British Journal of Psychiatry, 152, 799-806.

Cox, J. L., Holden, J. M., \& Sagovsky, R. (1987). Detection of postnatal depression. Development of the 10-item Edinburgh Postnatal Depression Scale. British Journal of Psychiatry, 150, 782-786.

Dennis, C. -L. (2004). Can we identify mothers at risk for postpartum depression in the immediate postpartum period using the Edinburgh Postnatal Depression Scale? Journal of Affective Disorders, 78, 163-169.

Dhawan, J., Bray, C. L., Warburton, R., Ghambhir, D. S., \& Morris, J. (1994). Insulin resistance, high prevalence of diabetes, and cardiovascular risk in immigrant Asians. Genetic or environmental effect? British Heart Journal, 72, 413-421.

Enas, E. A., \& Mehta, J. (1995). Malignant coronary artery disease in young Asian Indians: Thoughts on pathogenesis, prevention, and therapy. Coronary artery disease in Asian Indians (CADI) Study. Clinical Cardiology, 18, 131-135.

Enas, E. A., Yusuf, S., \& Mehta, J. L. (1992). Prevalence of coronary artery disease in Asian Indians. American Journal of Cardiology, 70, 945-949.

Gotlib, I. H., Whiffen, V. E., Mount, J. H., Milne, K., \& Cordy, N. I. (1989). Prevalence rates and demographic characteristics associated with depression in pregnancy and the postpartum. Journal of Consulting and Clinical Psychology, 57, 269-274.

Griffiths, R. (1976). The abilities of young children. High Wycombe, UK: The Test Agency.

Hales, C. N., \& Barker, D. J. (1992). Type 2 (non-insulindependent) diabetes mellitus: The thrifty phenotype hypothesis. Diabetologia, 35, 595-601.

Hales, C. N., Barker, D. J., Clark, P. M., Cox, L. J., Fall, C., Osmond, C., \& Winter, P. D. (1991). Fetal and infant growth and impaired glucose tolerance at age 64. British Medical Journal, 303, 1019-1022. 
McKeigue, P. M., Shah, B., \& Marmot, M. G. (1991). Relation of central obesity and insulin resistance with high diabetes prevalence and cardiovascular risk in South Asians. Lancet, 337, 382-386.

Mukhopadhyay, B., Forouhi, N. G., Fisher, B. M., Kesson, C. M., \& Sattar, N. (2006). A comparison of glycaemic and metabolic control over time among South Asian and European patients with Type 2 diabetes: Results from follow-up in a routine diabetes clinic. Diabetic Medicine, 23, 94-98.

Murray, L., \& Carothers, A. D. (1990). The validation of the Edinburgh Post-natal Depression Scale on a community sample. British Journal of Psychiatry, 157, 288-290.

Neale, M. C., Boker, S. M., Xie, G., \& Maes, H. H. (2002). Mx: Statistical modeling. Richmond: Department of Psychiatry.

Neel, J. V. (1962). Diabetes mellitus: A 'thrifty' genotype rendered detrimental by 'progress'? American Journal of Human Genetics, 14, 353-362.

O’Hara, M. W., Neunaber, D. J., \& Zekoski, E. M. (1984). Prospective study of postpartum depression: Prevalence, course, and predictive factors. Journal of Abnormal Psychology, 93, 158-171.

O’Keeffe, M. J., O’Callaghan, M., Williams, G. M., Najman, J. M., \& Bor, W. (2003). Learning, cognitive, and attentional problems in adolescents born small for gestational age. Pediatrics, 112, 301-307.

Ong, K. K., Ahmed, M. L., Emmett, P. M., Preece, M. A., \& Dunger, D. B. (2000). Association between postnatal catch-up growth and obesity in childhood: Prospective cohort study. British Medical Journal, 320, 967-971.

Prentice, A. M., Rayco-Solon, P., \& Moore, S. E. (2005). Insights from the developing world: Thrifty genotypes and thrifty phenotypes. Proceedings of the Nutrition Society, 64, 153-161.

Primatesta, P., \& Hirani, V. (2001). Anthropometry. In B. Erens, P. Primatesta, \& G. Prior (Eds.), Health survey for England: The health of minority ethnic groups '99. London: Joint Health Surveys Unit.
Ramsay, M., \& Fitzhardinge, P. (1977). A comparative study of two developmental scales: The Bayley and the Griffiths. Early Human Development, 1, 151-157.

Ray, J. G., Vermeulen, M. J., Shapiro, J. L., \& Kenshole, A. B. (2001). Maternal and neonatal outcomes in pregestational and gestational diabetes mellitus, and the influence of maternal obesity and weight gain: The DEPOSIT study. QJM, 94, 347-356.

Tolsa, C. B., Zimine, S., Warfield, S. K., Freschi, M., Sancho Rossignol, A. N. A., Lazeyras, F., Hanquinet, S., Pfizenmaier, M., \& Huppi, P. S. (2004). Early alteration of structural and functional brain development in premature infants born with intrauterine growth restriction. Pediatric Research, 56, 132-138.

Valdez, R., Athens, M. A., Thompson, G. H., Bradshaw, B. S., \& Stern, M. P. (1994). Birthweight and adult health outcomes in a biethnic population in the USA. Diabetologia, 37, 624-631.

Wallace, J. M., Bourke, D. A., Aitken, R. P., Milne, J. S., \& Hay, W. W., Jr. (2003). Placental glucose transport in growth-restricted pregnancies induced by overnourishing adolescent sheep. Journal of Physiology, 547(Pt 1), 85-94.

Wilcox, M., Gardosi, J., Mongelli, M., Ray, C., \& Johnson, I. (1993). Birth weight from pregnancies dated by ultrasonography in a multicultural British population. British Medical Journal, 307, 588-591.

Wu, G., Bazer, F. W., Cudd, T. A., Meininger, C. J., \& Spencer, T. E. (2004). Maternal Nutrition and Fetal Development. Journal of Nutrition, 134, 2169-2172.

Yajnik, C. S., Lubree, H. G., Rege, S. S., Naik, S. S., Deshpande, J. A., Deshpande, S. S., Joglekar, C. V., \& Yudkin, J. S. (2002). Adiposity and hyperinsulinemia in Indians are present at birth. Journal of Clinical Endocrinology \& Metabolism, 87, 5575-5580.

Yarbrough, D. E., Barrett-Connor, E., Kritz-Silverstein, D., \& Wingard, D. L. (1998). Birth weight, adult weight, and girth as predictors of the metabolic syndrome in postmenopausal women: the Rancho Bernardo Study. Diabetes Care, 21, 1652-1658. 